Nonlinear Processes in Geophysics, 12, 775-782, 2005

SRef-ID: $1607-7946 / \mathrm{npg} / 2005-12-775$

European Geosciences Union

(c) 2005 Author(s). This work is licensed

under a Creative Commons License.

\title{
A Kalman filter application to a spectral wave model
}

\author{
J. P. Pinto ${ }^{1}$, M. C. Bernardino ${ }^{1}$, and A. Pires Silva ${ }^{2}$ \\ ${ }^{1}$ Marinha, Instituto Hidrográfico, Portugal \\ ${ }^{2}$ Instituto Superior Técnico, Lisbon, Portugal
}

Received: 7 September 2004 - Revised: 22 July 2005 - Accepted: 27 July 2005 - Published: 9 August 2005

Part of Special Issue "Quantifying predictability"

\begin{abstract}
A sequential time dependent data assimilation scheme based on the Kalman filter is applied to a spectral wave model. Usually, the first guess covariance matrices used in optimal interpolation schemes are exponential spreading functions, which remain constant. In the present work the first guess correlation errors evolve in time according to the dynamic constraints of the wave model. A system error noise is deduced and used to balance numerical errors.

The assimilation procedure is tested in a standard situation of swell propagation, where the Kalman filter is used to assimilate the significant wave height. The evolution of the wave field is described by a linear two-dimensional advection equation and the propagation of the error covariance matrix is derived according to Kalman's linear theory.

Model simulations were performed in a 2-dimensional domain with deep-water conditions, a relatively small surface area and without wind forcing or dissipation. A true state simulation and a first guess simulation were used to illustrate the assimilation outcome, showing a reasonable performance of the Kalman filter.
\end{abstract}

\section{Introduction}

The availability of third-generation wave models and the increasingly capacity of oceanographic satellites to provide accurate measurements of altimeter data and, more recently, valuable directional information on the two-dimensional wave spectrum, have contributed substantially to the development of wave data assimilation. It is now clear that the assimilation methodologies can improve the sea state description both analysed and wave model forecasts, especially in swell dominated systems.

The early schemes developed for wave data assimilation were based on optimal interpolation (OI) methods, that combined significant wave height, mean period and directional

Correspondence to: M. C. Bernardino (mariana.bernardino@hidrografico.pt) data for updating the wave spectrum (Thomas, 1988; Esteva, 1988; Janssen et al., 1989, Lionello et al., 1992, 1995). These are computational efficient methods and consequently they have been tested in operational forecasting, using mainly altimeter data. However, the fact that forecast errors may be non-uniformly distributed over the wave spectrum restricts the enhancement achieved by wave height assimilation alone. Some authors have made an effort to overcome this limitation by merging altimeter and synthetic aperture radar (SAR) data (Hasselmann et al., 1997). In particular, a generalisation of the simple OI scheme was developed by applying a technique of spectral partitioning that allows to decompose the spectrum into a relatively small number of wave systems, each characterized by three integral spectral parameters (Gerling, 1992; Voorrips et al., 1997). The optimal interpolation schemes fit in the group of time independent assimilation methods. They simply update the model first guess by adding to it a weighted linear combination of the errors between the model and the observations. The covariance matrix of the observational and model errors determines the interpolation weights. Being time independent, the model correlation errors do not take into account the dynamic constraints of the model, which is a major disadvantage of this method.

In what concerns time dependent methods two main classes can be considered: sequential data assimilation methods and variational methods. The latter have been implemented in wave models using adjoint techniques (De Valk and Calkoen, 1989; de las Heras et al., 1992, 1994) or a Green function method (Bauer et al., 1996). These variational methods have the benefit of avoiding the problem of specifying the time history for the correlation errors of the model and observation data. But if the intent is to achieve an operational mode, several runs of the model are required to perform the variational procedure, leading to the need of large computational resources, as opposed to sequential methods.

In this paper, a sequential time dependent data assimilation scheme based on the Kalman filter is applied to a spectral wave model. This assimilation method should be a natural 
step forward from the optimal interpolation scheme. The main innovation is the introduction of time dependent correlation errors, which evolve according to the dynamic constraints of the model. The assimilation procedure is tested in a standard situation of swell propagation and the Kalman filter is used to assimilate the significant wave height, or more precisely the surface elevation variance. In the present case, the evolution of the wave field is described by a linear twodimensional advection equation and the propagation of the error covariance matrix is derived according to Kalman's linear theory.

\section{The wave model}

Stochastic or spectral models describe the propagation, growth and dissipation of wind gravity waves in large scales. The sea state is characterised by the spectral density action $N(t, x, y ; \sigma, \theta)$, a function of time, surface coordinates, relative frequency and direction. The associated spectral balance equation is given by

$$
\begin{aligned}
& \frac{\partial N}{\partial t}+\frac{\partial}{\partial x}\left(c_{x} N\right)+\frac{\partial}{\partial y}\left(c_{y} N\right)+\frac{\partial}{\partial \theta}\left(c_{\theta} N\right)+\frac{\partial}{\partial \sigma}\left(c_{\sigma} N\right)= \\
& \frac{S(N)}{\sigma}
\end{aligned}
$$

where the first term represents local time variations, the second and third term space propagation, the fourth the refraction essentially induced by bottom variations in space and the fifth term the Doppler shift induced by the presence of currents. The quantities $c_{x}, c_{y}, c_{\theta}$ and $c_{\sigma}$ represent the velocities in geographic space, direction space and frequency space, respectively. The functional $S(N)$ describes all the relevant energy transfer processes, and is generally decomposed into four terms

$S=S_{w g}+S_{d i s s}+S_{n l}+S_{b o t}$

namely, the wind wave growth, the white capping and wave breaking dissipation, non-linear wave interactions and bottom dissipation.

The wave parameters, such as the significant wave height, the mean period and the mean direction are obtained from spectral averaging. In particular, the significant wave height $\left(H_{S}\right)$ is defined as

$$
H_{s}=4\left(\int F d \sigma d \theta\right)^{1 / 2}
$$

where $F(t, x, y ; \sigma, \theta)$ is the wave spectral energy, related to the spectral density action according to

$N=\frac{F}{\sigma}$

If currents (tidal or others origins) are not considered, Eq. (1) can be written in a simpler form in terms of the wave spectral energy. It can be shown that

$$
\frac{\partial F}{\partial t}+\frac{\partial}{\partial x}\left(c_{x} F\right)+\frac{\partial}{\partial y}\left(c_{y} F\right)+\frac{\partial}{\partial \theta}\left(c_{\theta} F\right)=S(F)
$$

A detailed presentation of the theory can be found in Whitman (1974) and Komen et al. (1994), along with explicit expressions for the velocities.

Specifying an equation for the significant wave height correlations errors is essential to understand how this wave property evolves in time. This can be accomplished by integrating Eq. (5) in frequency and direction space, obtaining

$$
\frac{\partial \Psi}{\partial t}+\frac{\partial}{\partial x}\left(\bar{c}_{x} \Psi\right)+\frac{\partial}{\partial y}\left(\bar{c}_{y} \Psi\right)=16 \int S(F) d \sigma d \theta
$$

where $\Psi=H_{s}^{2}$ is the surface elevation variance, which is proportional to the wave energy (hereafter referred to as energy or wave energy). The velocity components fields $\bar{c}_{x}, \bar{c}_{y}$ are given by

$\bar{c}_{i}=\frac{\int c_{i} F d \sigma d \theta}{\int F d \sigma d \theta} \quad i=x, y$

The non-linear wave interactions are an internal energy transfer mechanism, conserving energy over the whole spectrum. The integration of this term in Eq. (6) is therefore zero. The remaining source terms are assumed to be reasonably well described by their first order approximation, resulting in a quasi-linear dependence on $F$

$S(F)=\alpha(F) F$

The proportionality factor $\alpha$ is a functional of $F$ depending on integrals over the spectra and on frequency and direction. This applies to the strong non-linear processes of whitecapping, dissipation by bottom friction and Miles wind wave generation mechanism. Defining

$\bar{\alpha}=\frac{\int \alpha(F) F d \sigma d \theta}{\int F d \sigma d \theta}$

and taking into account Eq. (8), one can rewrite Eq. (6) as

$\frac{\partial \Psi}{\partial t}+\frac{\partial}{\partial x}\left(\bar{c}_{x} \Psi\right)+\frac{\partial}{\partial y}\left(\bar{c}_{y} \Psi\right)=\bar{\alpha} \Psi$

This quasi-linear two-dimensional advection equation, with source terms, describes the evolution of the wave energy. It has a decisive importance to the Kalman filter application, as it prescribes a way to derive the time history of the energy correlation errors. The sea state evolution is still given by a full spectral calculation and in particular the velocity field and the coefficient $\bar{\alpha}$, which are essential to solve Eq. (10), always require the computation of the spectral Eq. (5).

Although in the setting up of Eq. (10) no explicit assumptions have been made about the form of the spectra, this scheme for handling energy correlation errors should only be totally valid for one-peaked spectra, corresponding to one wave system. If the sea state is characterized by a multipeaked spectra (e.g. crossing of two separate swell systems or a swell propagation over a local wind sea system) Eq. (10) would certainly fail to reproduce the correct time behaviour of the correlation errors since these different wave systems are uncorrelated. The complete specification of the covariance matrix would bear an impossible cost in practical applications. Yet, in real seas the coexistence of more than one 
wave system is not unusual. A way to circumvent this restriction is to split the spectra accordingly and assuming that each wave system evolves independently with no correlation. $\mathrm{Ne}-$ glecting non-linear interactions between different wave systems would then permit Eq. (10) to be applied to each partition. Naturally, the integrals in Eqs. (7) and (9) would also have to be taken over each partition, defining a velocity and a source coefficient for each wave system. However, this generalisation is only effective if the assimilated measurements identify and capture each wave system. Clearly this is not the case when one uses altimeter data.

\section{The assimilation scheme}

The sequential assimilation methods are usually called single time level schemes. Depending on the availability of measurements, the corrections to the model are made in a series of time frames centred at a certain time $t_{i}$. Contrary to variational methods, the field evolution is not entirely consistent with the model dynamics because a discontinuity is introduced at each assimilation time. Also the success of sequential data assimilation depends strongly on the reliability of the model first guess. On the other hand, sequential assimilation has a clear advantage for operational implementation since the model only has to run once.

The Kalman filter has been used extensively in oceanography (Ghil and Malanotte-Rizzoli, 1991; Evensen, 1992, 1993) and a complete derivation can be found in Jazwinski (1970). Here the focus is the application of the Kalman filter to a spectral wave model. Assuming that the energy field is represented at each given time $t_{k}$ by the $\mathrm{N}$-dimensional vector $\Psi_{k}$, with $N=N_{x} N_{y}$, where $N_{x}$ and $N_{y}$ are the number of grid points in the $x$ and $y$ direction, respectively, its evolution in time can be formulated as

$\Psi_{k}^{f}=A \Psi_{k-1}^{a}$

where $A$ is a linear operator advancing the state vector $\Psi$ in time. The superscripts $f$ and $a$ stand for forecast and analysed estimate, respectively. The real system evolution is given by

$\Psi_{k}^{t}=A \Psi_{k-1}^{t}+\Delta_{k}$

where $\Psi_{k}^{t}$ is the true state vector at $t_{k}$, and $\Delta_{k}$ is the system error originating from numerical approximations, unsatisfactory boundary conditions and deficient theoretical assumptions. The model correlation errors at $t_{k}$ are defined as

$P_{k}^{a, f}=\left\langle\left(\Psi_{k}^{a, f}-\Psi_{k}^{t}\right)\left(\Psi_{k}^{a, f}-\Psi_{k}^{t}\right)^{T}\right\rangle$

where \langle\rangle stands for the expected value and ()$^{T}$ for the transpose. The equation for the propagation of the model correlation errors is then obtained from Eqs.(11)-(12) as

$P_{k}^{f}=A P_{k-1}^{a} A^{T}+Q_{k}$

where $Q_{k}=\left\langle\Delta_{k} \Delta_{k}^{T}\right\rangle$ is the system error covariance.
The operator $A$ is derived from Eq. (10) and depends on the numerical method used. Since the present work only takes into account the propagation terms, a first order upwinding scheme was chosen. Although first order schemes have a larger numerical diffusion, compared to second order schemes, they are simpler to implement and require less computer resources. Considering this is an explicit integration and to fulfil numerical stability, the time step has to satisfy the inequality $c \leq 1$, where $c$ is the CFL number given by $\Delta t\left(\bar{c}_{x} / \Delta x+\bar{c}_{y} / \Delta y\right)$. As explained before, Eq. (5) has to lead the computation suite in order to provide the velocity field and the factor $\alpha$, used in the iteration rule of Eq. (14).

For the energy correlation error, the assimilation time step is larger than the computation time step. Therefore, between assimilation points several iterations of Eq. (14) could be made. In that case superscript $a$ should be replaced by $f$. At the assimilation time, the measurements available at some points and can be related to the true state through the equation

$\Psi_{k}^{o}=L_{k} \Psi_{k}^{t}+\delta_{k}$

where the $N_{o b s}$ dimensional vector $\Psi_{k}^{o}$ represents the observation state ( $N_{o b s}$ is the number of assimilation points) and $\delta_{k}$ is the measurement error term. The $N_{o b s} \times N$ matrix $L_{k}$ projects the model space into the observational space. The analysed estimate field is obtained according to

$\Psi_{k}^{a}=\Psi_{k}^{f}+K_{k}\left(\Psi_{k}^{o}-L_{k} \Psi_{k}^{f}\right)$

where the $N \times N_{o b s}$ Kalman gain matrix is given by

$K_{k}=P_{k}^{f} L_{k}^{T}\left(L_{k} P_{k}^{f} L_{k}^{T}+O_{k}\right)^{-1}$

and $O_{k}=\left\langle\delta_{k} \delta_{k}^{T}\right\rangle$ is the observation error covariance $N_{o b s} \times N_{o b s}$ matrix. The Kalman matrix is able to scatter the assimilation improvement to all grid points, a process dominated by the spatial scales inherent to the model correlation errors and by a compromise between model errors and observation errors. These scales evolve dynamically according to the model constraints.

The observation error matrix $\mathbf{O}$ must be specified at each assimilation time. Assuming uncorrelated measuring instruments

$\mathbf{O}_{i j}=\delta_{i j} R_{i} i, j=1, \ldots, N_{o b s}$

where $\delta_{i j}$ is the Kronecker symbol and $R_{i}$ is the observation error variance associated with assimilation point $i$.

When the filter is applied to obtain an analysed estimate, the model error is reduced and updated according to

$P_{k}^{a}=P_{k}^{f}-K_{k} L_{k} P_{k}^{f}$

However, as noted by Evensen (1992), this expression may become negative due to numerical errors in regions of low variance. To overcome this problem the equivalent equation is used

$P_{k}^{a}=\left(I-K_{k} L_{k}\right) P_{k}^{f}\left(I-K_{k} L_{k}\right)^{T}+K_{k} O_{k} K_{k}^{T}$, 
which is given by the sum of two positive defined matrices.

Before running Eq. (5) for the next time step, the wave spectra has to be updated and a correction is introduced in the model through Eq. (16). The simplest way for the new spectra to match the analysed energy is to consider the following equation

$$
F^{a}(\sigma, \theta)=T F^{f}(\sigma, \theta) \quad T=\frac{\Psi^{a}}{\Psi^{f}}
$$

at each grid point, where $F^{a}$ and $F^{f}$ are the analysed and the forecast spectra, respectively. The consistency of this choice is discussed in Janssen et al. (1989) and Lionello et al. (1992) and it is clear that this solution is only suitable when no major feature of the true spectrum is absent in the model estimate.

For the test case studied in this article, the initial and the incoming boundary conditions of the correlation errors are defined according to

$$
P_{i j}^{f}=\sigma_{i}^{f} \sigma_{j}^{f} \exp \left(-\frac{d_{i j}}{D}\right) \quad i, j=1, \ldots, N
$$

where

$$
\sigma_{i}^{f}=\left\langle\left(\Psi_{i}^{f}-\Psi_{i}^{t}\right)^{2}\right\rangle^{1 / 2}
$$

is the root-mean-square model error at grid point $i$ and $d_{i j}$ is the distance between the grid points $i$ and $j$. The parameter $D$ is a constant correlation length scale. The outgoing boundary is treated as an open boundary.

The root-mean-square (Eq. 23) is expressed with a parameterisation obtained from a statistical analysis of comparisons between model predictions and observations off the Portuguese coast, namely

$\sigma^{f}=\frac{1}{(1+\varepsilon)^{1 / 2}}\left(0.096+0.124 H_{s}\right)$.

$\varepsilon$ is an adjustable parameter defined as

$\varepsilon^{1 / 2}=\frac{\sigma^{o}}{\sigma^{f}}$

The deduction of Eq. (24) follows Voorrips et al. (1997) where it was assumed that the model estimate errors and the observation errors are uncorrelated. In particular, Eq. (25) implies that the observation error variance is given by

$$
R_{i}=\varepsilon\left(\sigma_{i}^{f}\right)^{2}
$$

This equation is used throughout the assimilation procedure with the purpose of evaluating the observation error matrix O.

The time iteration for correlation matrix $\mathbf{P}$ implies at each step the knowledge of the system noise covariance $Q$, which depends on several inherent errors. In the Kalman framework, the calculation of the system noise $Q$ is initiated from some random distribution with zero average. Nevertheless, to explicitly evaluate the $\mathbf{Q}$ matrix some assumptions, which depend on the type of model errors one tries to minimise
(Zang and Malanotte-Rizzoli, 2003), have to be made. In order to balance numerical diffusion analysis, an expression for the system noise error is deduced (see Appendix A),

$$
Q_{k}=\left(\exp \left(\frac{\Delta s}{D}\right)-1\right) D g\left(A \bar{P}_{k-1} A^{T}\right)
$$

where $\bar{P}$ is the off-diagonal part of the model covariance errors covariance, $D g$ () stands for the diagonal part and $\Delta s$ for grid point spacing. According to Eq. (27) only the variance field is affected directly by this correction. At each grid point it is given by the correlation error times $2 c(1-c)$, summed over the closest upwind grid points. The exponential factor includes both the spatial resolution and the correlation scale so that $Q_{k} \rightarrow 0$ when $\Delta s \rightarrow 0$ or $D \rightarrow+\infty$. The expression (27), deduced to match numerical diffusion, has only two parameters. One is the CFL number $c$, which depends on the time step, the grid spacing and the velocity and should always be close to one for numerical stability. The other is the scaling parameter $D$ from expression (22). As there is no sound rule for prescribing the system noise matrix, the reliability of expression (27) can only be validated by its results.

\section{Results}

A 2-dimensional domain, with deep-water conditions but a relatively small surface area $(400 \mathrm{~km} \times 600 \mathrm{~km})$, was adopted. The corresponding number of grid points is $81 \times 121$. At this stage, the main goal is to understand and analyse the model correlation errors evolution and the filter performance in a simple case of swell propagation. For this reason, the wind forcing and the dissipation mechanisms were not included in Eqs. (5) and (14). In that case, the wave model has to be solved as a boundary value problem and wave conditions (significant wave height, peak period and wave direction) must be specified along the incoming boundaries. The incoming significant wave height is also used to define the boundary conditions for the energy correlation errors, according to Eqs. (22) and (24). In Table 1 the wave parameters defining the boundary conditions for the true state and the model state at the left top corner of the computation domain are presented. They replicate the arrival of two comparable wave systems each having a swell event around day 4 . Finally, the free parameters $D$ and $\varepsilon$ were fixed for the whole set of simulations with the values $D=60 \mathrm{~km}$ and $\varepsilon=0.2$.

A stationary run of Eq. (5) is needed to estimate an initial condition field. At each time step, the velocities are calculated using Eq. (7) and subsequently the operator A is determined. The model correlation errors are then solved for the next time step according to Eq. (14). As soon as the process of assimilation is done, energy observations $\Psi^{o}=H_{S}^{2}$ (taken from the true state simulation) are used to obtain the analysed energy field following Eq. (16). The correlation errors and the wave spectra field are also corrected through Eqs. (20) and (21), respectively.

To test the behaviour of the correlation errors time iteration, Eq. (14) two simulations without assimilation have been 
Table 1. Boundary conditions for the true state and the model state.

\begin{tabular}{ccccccc}
\hline Time & \multicolumn{3}{c}{ True state } & \multicolumn{3}{c}{ Model state } \\
& $H_{S}$ & $T_{p}$ & $D_{i r}$ & $H_{s}$ & $T_{p}$ & $D_{i r}$ \\
\hline 00.00 & 2.61 & 8.83 & 301.7 & 3.28 & 7.61 & 297.2 \\
00.06 & 2.45 & 8.71 & 300.2 & 3.01 & 7.59 & 303.3 \\
00.12 & 2.21 & 8.77 & 304.7 & 2.55 & 7.34 & 305.6 \\
00.18 & 2.07 & 8.63 & 309.1 & 2.17 & 7.14 & 306.7 \\
01.00 & 1.98 & 7.71 & 311.6 & 1.92 & 7.09 & 300.5 \\
01.06 & 1.78 & 7.63 & 306.3 & 1.91 & 7.33 & 300.2 \\
01.12 & 1.58 & 7.81 & 308.8 & 2.03 & 7.81 & 299.6 \\
01.18 & 1.42 & 7.79 & 303.0 & 1.98 & 8.06 & 298.2 \\
02.00 & 1.44 & 8.00 & 304.7 & 1.89 & 6.15 & 299.3 \\
02.06 & 1.54 & 8.37 & 306.0 & 2.01 & 5.68 & 302.5 \\
02.12 & 1.56 & 7.22 & 305.1 & 1.94 & 5.84 & 297.6 \\
02.18 & 1.55 & 6.17 & 305.7 & 1.76 & 5.81 & 292.3 \\
03.00 & 1.58 & 6.20 & 303.4 & 1.82 & 6.19 & 287.9 \\
03.06 & 1.54 & 6.59 & 306.0 & 3.66 & 10.51 & 289.9 \\
03.12 & 1.55 & 7.81 & 299.7 & 5.34 & 11.81 & 288.7 \\
03.18 & 3.01 & 13.21 & 297.2 & 5.56 & 11.64 & 288.1 \\
04.00 & 4.29 & 13.77 & 295.8 & 5.36 & 11.20 & 289.8 \\
04.06 & 4.48 & 13.46 & 295.9 & 5.05 & 10.07 & 287.8 \\
04.12 & 4.31 & 13.20 & 296.5 & 5.09 & 8.96 & 290.3 \\
04.18 & 3.89 & 12.02 & 297.0 & 5.11 & 8.73 & 289.5 \\
05.00 & 3.63 & 10.94 & 297.7 & 4.77 & 8.52 & 293.0 \\
05.06 & 3.53 & 10.07 & 298.3 & 4.13 & 8.17 & 293.2 \\
05.12 & 3.38 & 9.76 & 300.1 & 3.47 & 7.96 & 293.1 \\
05.18 & 3.12 & 9.42 & 302.2 & 2.86 & 7.73 & 293.2 \\
06.00 & 2.78 & 9.04 & 303.1 & 2.39 & 7.47 & 294.8 \\
06.06 & 2.39 & 8.24 & 305.5 & 2.18 & 7.09 & 296.4 \\
06.12 & 2.08 & 7.69 & 300.6 & 2.35 & 7.04 & 280.0 \\
06.18 & 2.36 & 6.35 & 301.9 & 2.98 & 7.81 & 271.9 \\
07.00 & 3.70 & 7.34 & 321.5 & 2.91 & 7.72 & 271.1 \\
07.06 & 4.12 & 7.85 & 340.1 & 2.53 & 7.35 & 273.8 \\
07.12 & 3.63 & 7.48 & 300.7 & 2.15 & 7.35 & 281.9 \\
07.18 & 3.01 & 6.91 & 296.8 & 1.88 & 7.34 & 279.5 \\
08.00 & 2.53 & 6.43 & 292.3 & 1.67 & 7.34 & 274.9 \\
08.06 & 2.19 & 6.11 & 296.4 & 1.52 & 6.98 & 268.8 \\
08.12 & 1.88 & 5.69 & 280.7 & 1.52 & 6.45 & 274.2 \\
08.18 & 1.60 & 5.56 & 285.5 & 1.94 & 7.35 & 278.9 \\
09.00 & 1.37 & 5.96 & 281.9 & 2.67 & 8.82 & 282.3 \\
\hline & & & & & &
\end{tabular}

selected. The results obtained for the evolution of the model errors variance $\mathrm{P}$ over one day are presented in Figs. 1 and 2 , with and without the system noise error, respectively, as defined in Eq. (27). Both simulations start at the beginning of the swell event and each run shares the same boundary condition. In general, the computation time step for evaluating Eqs. (5) and (14) is less than one hour, satisfying the condition $c \approx 1$.

In the absence of a dynamical forcing, the variance errors should propagate without any significant change. Except for the small decrease expected to occur as the area occupied by the waves is increasing (in this particular, variance errors behave like the energy). The time frames in Fig. 1 indicate, however, a completely different behaviour of the variance errors propagation. The incoming swell causes a sudden and
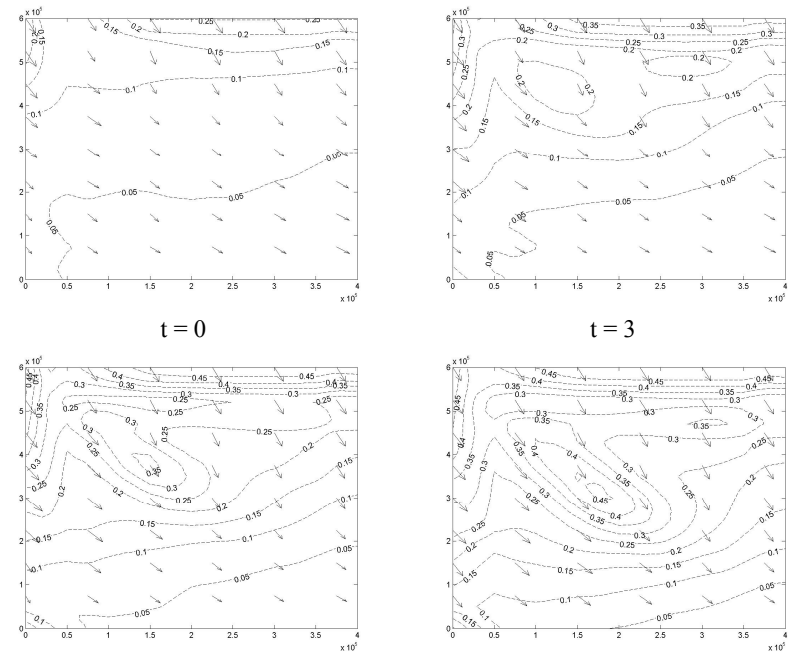

$\mathrm{t}=3$

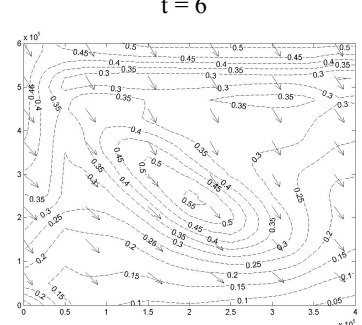

$\mathrm{t}=12$
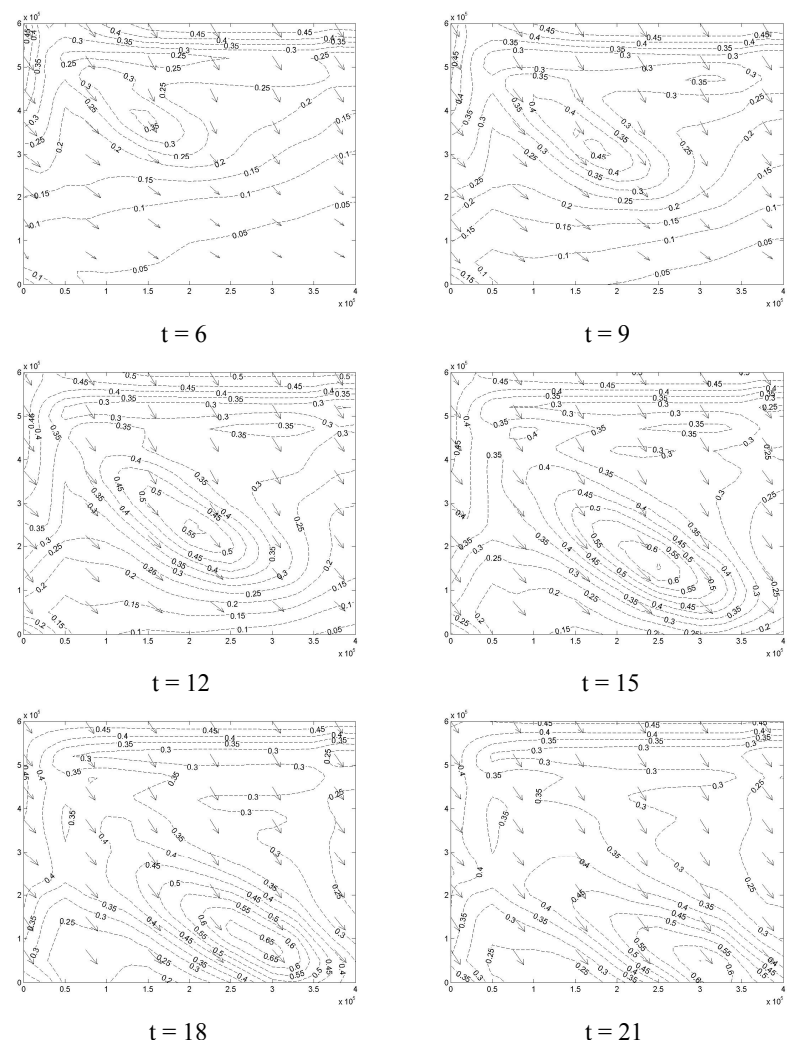

$=9$

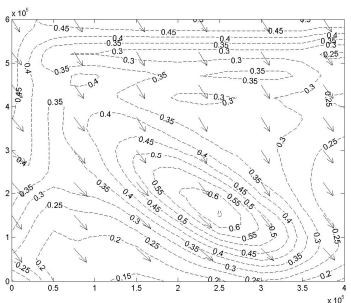

$\mathrm{t}=15$

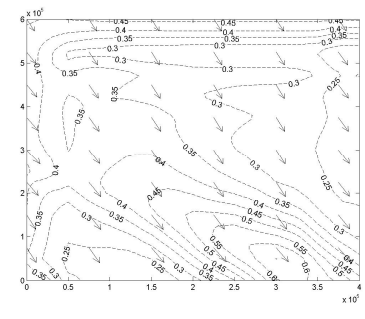

$\mathrm{t}=21$

Fig. 1. Model error variance $\left(\mathrm{m}^{2}\right)$ and group velocity field $(\mathrm{m} / \mathrm{s})$ at different time levels, excluding the system noise error. The parameters' values $D=60 \mathrm{~km}$ and $\varepsilon=0.2$ were used in this simulation.

fast increase in the variance field at the boundary, leading to an enhanced numerical diffusion that generates a growing peak inside the domain. As shown in Fig. 2, the introduction of the system noise given by Eq. (27) balances this extraneous effect. As a consequence, a smoother and more satisfactory propagation of the variance errors is achieved. The comparison shows that numerical errors could be reasonably large, creating discrepancies in the order of $20 \%$. Evensen (1992) had already pointed out that the numerical diffusion associated with the advection scheme can cause a significant decrease of the variance field in regions of high velocity. The use of a less diffusive scheme and higher resolution could provide better results for the correlation error propagation but this would increase excessively the computation time. Wider covariance functions (large values of $D$ ) would also decrease the diffusion but at the expense of introducing unrealistic and non-physical correlation scales. 

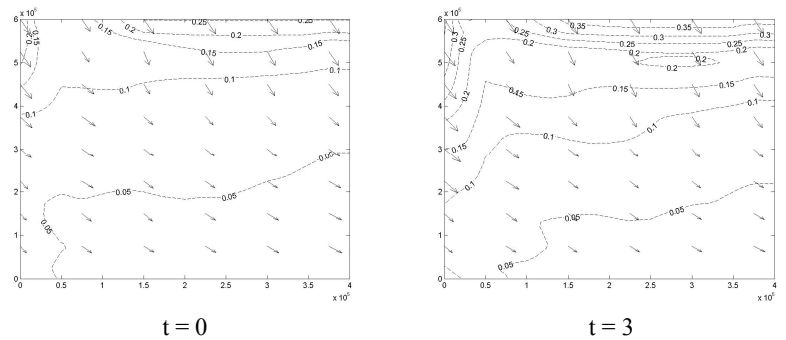

$\mathrm{t}=3$

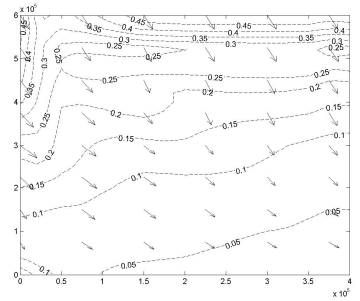

$t=6$

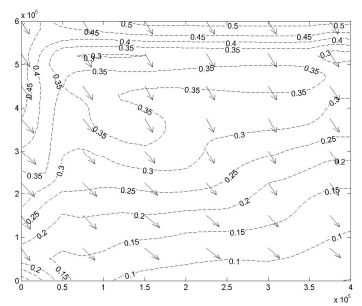

$\mathrm{t}=12$

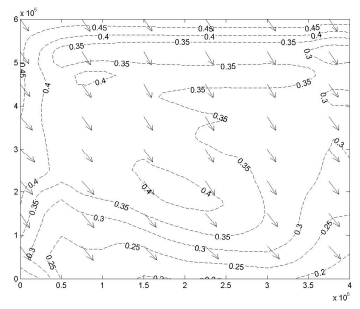

$\mathrm{t}=18$

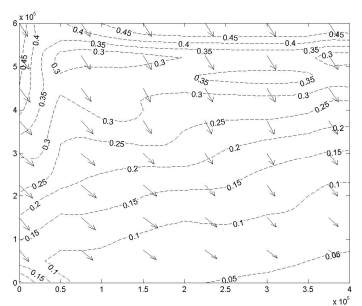

$t=9$

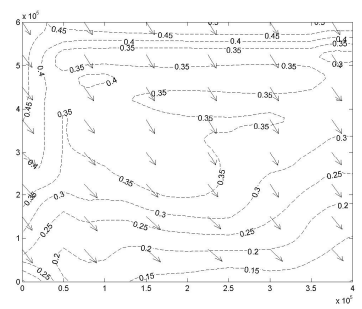

$t=15$

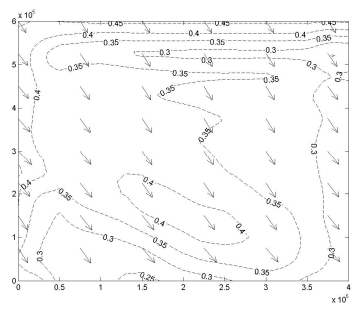

$\mathrm{t}=21$
Fig. 2. Same as Fig. 1, but including the system noise error.

The three simulations presented hereafter aim to assess the filter performance. The first one will be regarded as the true state. The second one reproduces the model estimate with no assimilation and the third one incorporates the assimilation procedure in the second simulation. All these simulations include the system noise error. Since this example is solved as a boundary value problem, the simulations were performed with two close boundary conditions (see Table 1), one for the true state and another for the model state.

The assimilation is carried out by inserting at two points the respective true state values, taken here as the observations values. The justification for this procedure lies in the fact that "the true state" is merely a device for assessing the filter's performance.

The assimilation effect on the observation points is depicted in Fig. 3. The top figure shows results obtained at coordinates $(50,400)(\mathrm{km})$ and the bottom figure the results obtained at coordinates $(150,500)(\mathrm{km})$. The assimilation time step is $6 \mathrm{~h}$. The analysis converges to the true state, but
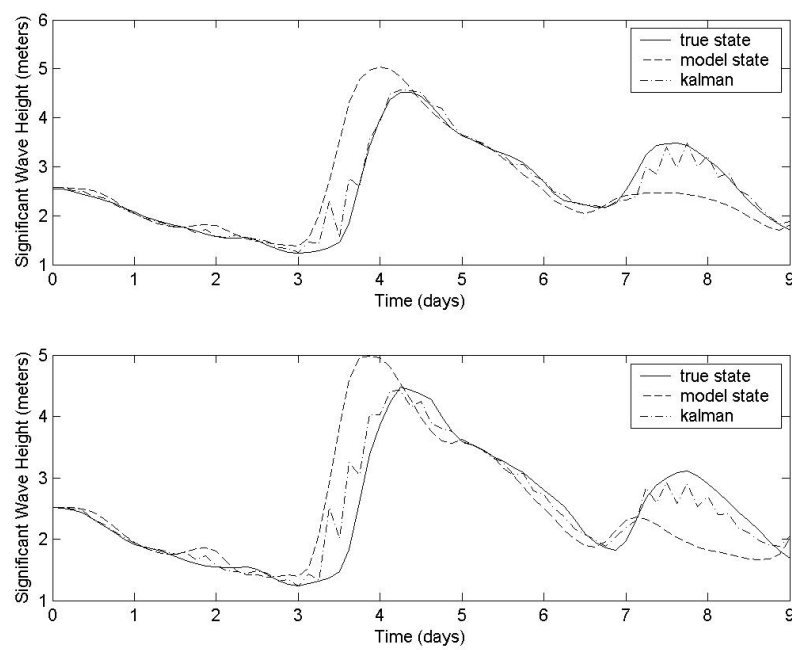

Fig. 3. Time history for the significant wave height at the assimilation points. The solid line and the dashed line represent the true state and the model state, respectively. The dot dash line represents the analysed state obtained in the course of the assimilation process based on the Kalman filter and in the following $6 \mathrm{~h}$ forecast.

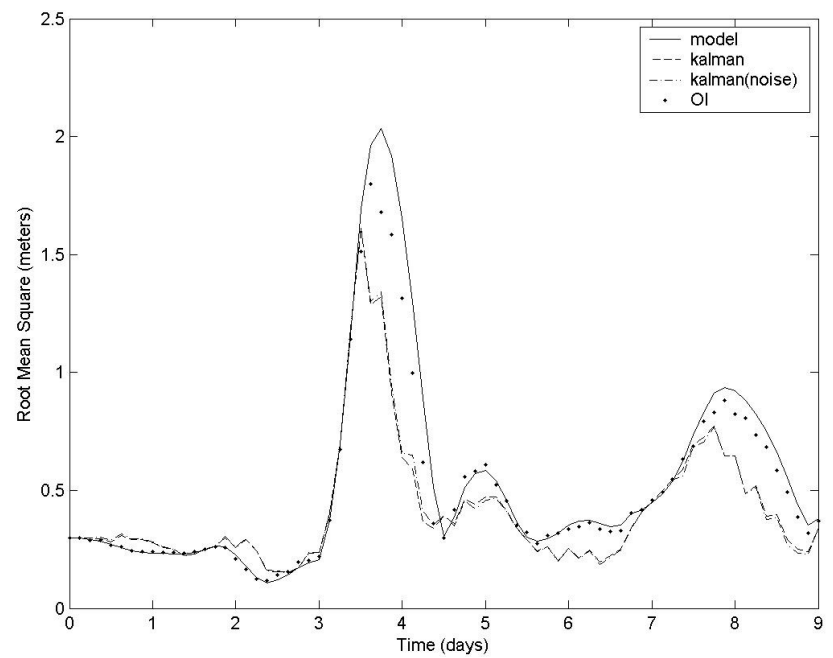

Fig. 4. Energy root mean square difference between the true state and the model state (solid line), the true state and the Kalman filter simulation with no noise (dashed line), the true state and the Kalman filter simulation with noise (dash dot line) and the true state and the optimal interpolation assimilation scheme (dots).

this tendency is quickly inverted by the model boundary conditions influence during the time interval between successive assimilations. The swift hops between the analysis and the forecast are more significant when the difference among the true state and the model state increases, which is a typical behaviour of sequential methods whenever the conditions that control the model evolution are kept unchanged after the assimilation.

To have a global evaluation of the filter's performance, the root mean square (hereafter RMS) differences between the true state and the model predictions (with and without 
assimilation) are computed at each time step and shown in Fig. 4. It can be seen that there is a generalized reduction of the RMS differences when the Kalman filter is used and that this reduction is stronger when the mismatch is higher. But large RMS values during important events are still observed. These facts suggest that some features of the true state spectra cannot be reproduced by the model only by an energy renormalisation. Further improvements should require also the assimilation of the mean period and the subsequent spectra reconstruction.

The overall filter's performance is not sensitive to the inclusion of the system noise error. This could be explained by the location of the assimilation points, which were chosen close to the incoming boundary, where the smoothness introduced by the noise error is not substantial. Figures 1 and 2 show that the diffusion effect is more pronounced downward, hardly affecting the assimilation.

A simulation using the OI assimilation scheme is included additionally in order to compare its results with the Kalman filter. The optimal interpolation technique is also based on a sequential correction by combining the model estimate with its observed counterpart through the update Eq. (16). The weighting coefficients are given by the Kalman gain Eq. (17) too. However, the model correlation errors, computed according to Eq. (22), remain fixed at all times. The comparison between the Kalman filter and the OI scheme shows that the former exhibits a better overall behaviour, especially in events where the deviations from the true state are important.

\section{Conclusions}

A Kalman filter assimilation scheme has been implemented in an ocean wave model. A simple case of linear advection in a two-dimensional space, particularly suitable for the simulation of a well-developed swell, was tested. The wind-driven forcing and the dissipations mechanisms were ignored, implying that the correlation matrix depends just on the velocity field.

It was found that the numerical errors of the energy correlation errors time iteration could be large. To minimize the model covariance errors propagation to an acceptable level, an expression for the system noise was introduced. This correction is responsible for offsetting most of the numerical diffusion originated by the advection scheme, keeping the solution stable over the nine days during which the simulation took place. This way, the use of higher order numerical methods seems to be avoidable, simplifying considerably the computational approach.

A true state simulation and a first guess simulation were used to illustrate the assimilation results and the improvement brought by the Kalman filter. Another finding that comes out of the experiment is the insufficiency of assimilating only the Hs. The mean period and the average direction should be included in the assimilation structure as well. This inclusion would increase extensively the numerical handling of the Kalman filter.
The comparison between a first order scheme, considering noise error, and a less diffusive scheme without noise error is an interesting point to be addressed in future investigations. This would emphasize how the system noise, given by Eq. (27) and designed to balance the model discrepancies, would perform. Naturally, the analysis of a wind-driven case is the real judgement of this assimilation method. The inclusion of the wind forcing would show the Kalman filter handling the correlation scaling induced by the dynamics.

\section{Appendix A System noise error}

For simplicity, let us consider 1D propagation along three grid points, where grid point 1 is the incoming boundary. In that case, the advection operator A for the upwinding scheme is a $3 \times 3$ matrix given by

$A=\left[\begin{array}{ccc}1 & 0 & 0 \\ c & 1-c & 0 \\ 0 & c & 1-c\end{array}\right]$

where $c=c_{x} \Delta t / \Delta x$ is the CFL number, depending on the velocity $c_{x}$, the time step $\Delta t$ and the grid space. According to Eq. (14) and Eq. (A1) and neglecting the system noise error, the variance error at grid point 2 at time level $k$ is

$P_{2}^{k}=c^{2} P_{1}^{k-1}+2 c(1-c) P_{12}^{k-1}+(1-c)^{2} P_{2}^{k-1}$

where $P_{1}^{k-1}$ and $P_{2}^{k-1}$ are the variance error at time level $k-1$ in grid point 1 and 2 , respectively, and $P_{12}^{k-1}$ is the correlation error between grid point 1 and grid point 2 at time level $k-1$. If $c=1 \mathrm{Eq}$. (A2) states that $P_{2}^{k}=P_{1}^{k-1}$, which means that the variance propagates from grid point 1 to grid point 2, as expected. And yet, the Eq. (A2) does not produce in general a correct advection for $c \neq 1$. In fact, the propagation should follow the iteration rule

$P_{2}^{k}=c P_{1}^{k-1}+(1-c) P_{2}^{k-1}$

To get the right equation for the error variance propagation a quantity is added to the second member of Eq. (A2) to match the expected result, so

$$
\begin{aligned}
& c^{2} P_{1}^{k-1}+2 c(1-c) P_{12}^{k-1}+(1-c)^{2} P_{2}^{k-1}+Q_{2}^{k} \\
& =c P_{1}^{k-1}+(1-c) P_{2}^{k-1}
\end{aligned}
$$

from which results

$Q_{2}^{k}=c(1-c)\left(P_{1}^{k-1}-2 P_{1}^{k-1}+P_{2}^{k-1}\right)$

From this viewpoint, the diagonal matrix $\mathrm{Q}$ defined in Eq. (A5) is to be considered as a system noise error for dealing with the numerical diffusion present in the iteration scheme of the Eq. (A2). Defining the correlation error as $P_{12}^{k-1}=\sigma_{1}^{k-1} \sigma_{2}^{k-1} a_{12}^{k-1}$ and noting that the variances errors are given by $P_{1}^{k-1}=\left(\sigma_{1}^{k-1}\right)^{2}$ and $P_{2}^{k-1}=\left(\sigma_{2}^{k-1}\right)^{2}$, where 
$\sigma_{i}^{k-1}$ is the root-mean-square model error at grid point $i$ defined in Eq. (23), will allow equation (A5) to be rewritten in the equivalent form

$$
Q_{2}^{k}=c(1-c)\left(\left(\sigma_{1}^{k-1}-\sigma_{2}^{k-1}\right)^{2}+2 \sigma_{1}^{k-1} \sigma_{2}^{k-1}\left(1-a_{12}^{k-1}\right)\right)
$$

Neglecting the first term on the right hand side of Eq. (A6), which is relatively smaller, gives

$$
Q_{2}^{k}=2 c(1-c) P_{12}^{k-1}\left(\exp \left(\frac{\Delta x}{D}\right)-1\right)
$$

where an exponential decay was assumed for the correlation errors. The former expression can be written in terms of the advection operator $\mathrm{A}$,

$$
Q^{k}=\left(\exp \left(\frac{\Delta x}{D}\right)-1\right) D g\left(A \bar{P}^{k-1} A^{T}\right)
$$

where $D g()$ stands for diagonal part and $\bar{P}$ for the offdiagonal part of the model covariance errors. This reasoning can be worked out for a $2 \mathrm{D}$ propagation showing that Eq. (A8) is still valid in that situation.

Acknowledgements. This study is part of two research projects: PAMMELA2 (Nearshore Wave Prediction: Spectral Models and Data Assimilation) supported by grant PDCTM/P/MAR/15242/1999 from the Portuguese Foundation for Science and Technology (FCT) of the Ministry of Science and High Education and MOCASSIM ("Implementação de Modelos Oceanográficos com Assimilação de Dados”) supported by "Programa de Apoio aos Laboratórios do Estado do Ministério da Ciência e Tecnologia" and "Programa Operacional Ciência, Tecnologia e Inovação do Quadro Comunitário de Apoio III".

The authors wish to thank the anonymous referees for the valuable comments and suggestions.

Edited by: S. Vannitsem

Reviewed by: two referees

\section{References}

Bauer, E., Hasselmann, K., Young I., and Hasselmann, S.: Assimilation of wave data into an ocean wave model using an impulse response function method, J. Geophys. Res., 101, 3801-3816, 1996.

de las Heras, M. and Janssen, P. A. E. M.: Data assimilation with a non-linear coupled wind wave model, J. Geophys. Res., 97, $20261-20270,1992$. de las Heras, M., Burgers, G., and Janssen, P. A. E. M.: Variational wave data assimilation in a third-generation wave model, J. Atmos. Oceanic Thecnol., 11, 1350-1369, 1994.

De Valk, C. F. and Calkoen, C. J.: Wave data assimilation in a third generation wave prediction model for the North Sea - An optimal control approach, Delft Hydraul. Lab., The Netherlands, Rep. X38, 123 pp., 1989.

Esteva, D. C.: Evaluation of preliminary experiments assimilating Seasat significant wave heights into a spectral wave model, J. Geophys. Res., 93, 14 099-14 106, 1988.

Evensen, G.: Using the extended Kalman filter with a multiplayer quasi-geostrophic ocean model, J. Geophys. Res., 97, 17905$17924,1992$.

Evensen, G.: Open boundary conditions for the extended Kalman filter with a quasi-geostrophic ocean model, J. Geophys. Res., 98, 16529-16546, 1993.

Gerling, T.: Partitioning sequences and arrays of directional ocean wave spectra into component wave systems, J. Atmos. Oceanic Technol., 9, 444-458, 1992.

Ghil, M. and Malanotte-Rizzoli, P.: Data assimilation in meteorology and oceanography, Adv. Geophys., 33, 141-266, 1991.

Hasselmann, S., Lionello, P., and Hasselmann, K.: An optimal interpolation scheme for the assimilation of spectral wave data, J. Geophys. Res., 102, 15 823-15 836, 1997.

Janssen, P. A. E. M., Lionello, P., Reistad, M., and Hollingsworth, A.: Hindcasts and data assimilation studies with the WAM model during the Seasat period, J. Geophys. Res., 94, 973-993, 1989.

Jazwinski, A. H.: Stochastic Processes and Filtering Theory, Academic, San Diego, California, 1970.

Komen, G. J., Cavaleri, L., Donelan, M., Hasselmann, K., Hasselmann, S., and Janssen, P. A. E. M.: Dynamics and Modelling of Ocean Waves, Cambridge Univ. Press, New York, 1994.

Lionello, P., Günther, H., and Janssen, P. A. E. M.: Assimilation of altimeter data in a global third-generation wave model, J. Geophys. Res., 97, 14 453-14 474, 1992.

Lionello, P., Günther, H., and Hansen, B.: A sequential assimilation scheme applied to global wave analysis and prediction, J. Mar. Syst., 6, 87-107, 1995.

Thomas, J. P.: Retrieval of energy spectra from measured data for assimilation into a wave model, Q. J. R. Meteorol. Soc., 114, 781-800, 1988.

Voorrips, A. C., Makin, V. K., and Hasselmann, S.: Assimilation of wave spectra from pitch-and-roll buoys in a North Sea wave model, J. Geophys. Res., 102, 5829-5849, 1997.

Whitman, G. B.: Linear and non linear waves, Wiley, New York, 1974.

Zang, X. and Malanotte-Rizzoli, P.: A comparison of assimilation results from the ensemble Kalman Filter and a reduced-rank extended Kalman Filter, Nonlin. Processes Geophys., 10, 477-491, 2003, SRef-ID: 1607-7946/npg/2003-10-477. 